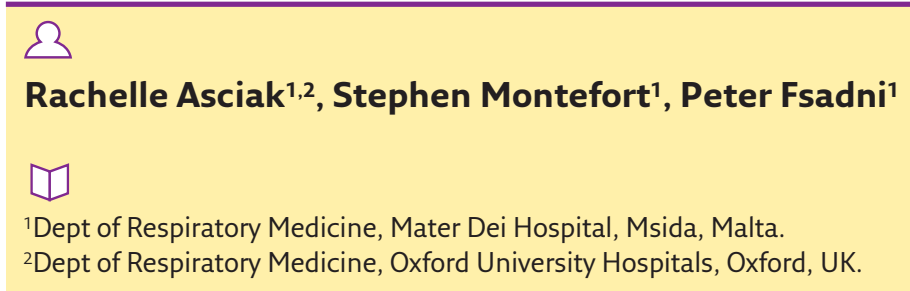

'Dept of Respiratory Medicine, Mater Dei Hospital, Msida, Malta

2Dept of Respiratory Medicine, Oxford University Hospitals, Oxford, UK.

\title{
Respiratory medicine training in Malta
}

In Malta, respiratory medicine is quite a popular speciality. It is attractive because of the interesting case mix and the interventional element of the speciality.

To understand the training system in Malta, it helps to first have some background knowledge of Malta. Malta is a tiny Mediterranean island, just $316 \mathrm{~km}^{2}$ in area, with one main teaching hospital, Mater Dei Hospital, with a second smaller hospital on the sister island of Gozo. Scattered across the island are regional health centres, and two smaller oncology and rehabilitation hospitals. These cater for the 420000 people living there. With tourism, the population increases significantly. We communicate in both Maltese and English, and teaching (both at medical school and at a postgraduate level) is in English.

\section{Entry into respiratory medicine}

After graduation from medical school, doctors spend 2 years in a foundation programme, and rotate through various specialities both in and out of the main hospital. At the end of the foundation programme, they apply for their preferred general specialities (called Basic Specialist Training). Aspiring respiratory physicians will need to apply for general internal medicine, and spend 2 years rotating through various subspecialties within general medicine, including respiratory medicine. During this period, they must also pass the Membership of the Royal College of Physicians (UK) postgraduate examinations. On obtaining a certificate of successful completion of basic/core training, doctors are eligible to apply for the post of respiratory medicine higher specialist trainee; these posts typically become available annually.

\section{Specialisation}

The Specialist Accreditation and Postgraduate Training Committees regulate the specialisation programme in respiratory medicine. This involves dual accreditation for general internal medicine and respiratory medicine. Core general medical trainees can progress to higher specialist training in General Internal Medicine and Respiratory. A general internal medicine Certificate of Completion of Training (CCT) is awarded after 3 years of full-time training while the respiratory CCT is awarded after the fourth year, provided the doctor has also passed the speciality certificate examination set by the Royal College of Physicians or the HERMES (Harmonised Education in Respiratory Medicine for European Specialists) examination set by the European Respiratory Society. Progression through the 4 years of training is based on obtaining the necessary competencies and annual review of competence progression results, as dictated by the training association. Once on the specialist register, the respiratory physician can work independently as a specialist in respiratory medicine and, provided they meet the necessary criteria, can apply for consultant posts as they are issued.

\section{Teaching}

Trainees are expected to attend and present at regular journal clubs, and guideline meeting where the latest guidelines are discussed and applied to daily practice. Additional monthly teaching is also held in the evenings. During higher specialist training, trainees are encouraged to spend a period of 1 year training overseas, usually in the UK, in order to broaden their knowledge and exposure.

An academic interest is encouraged, and several trainees opt for a part-time postgraduate degree, including Masters degrees or PhDs.

\section{Day-to-day practice}

Trainees are assigned to a consultant for a minimum 1-year period. A firm consists of a consultant, a respiratory medicine higher specialist trainee, a basic specialist trainee in general medicine and a foundation trainee doctor. The firm's weekly timetable includes daily ward rounds of inpatients,
Cite as: Asciak R, Montefort S, Fsadni P. Respiratory medicine training in Malta. Breathe 2017; 13: 245-246. 


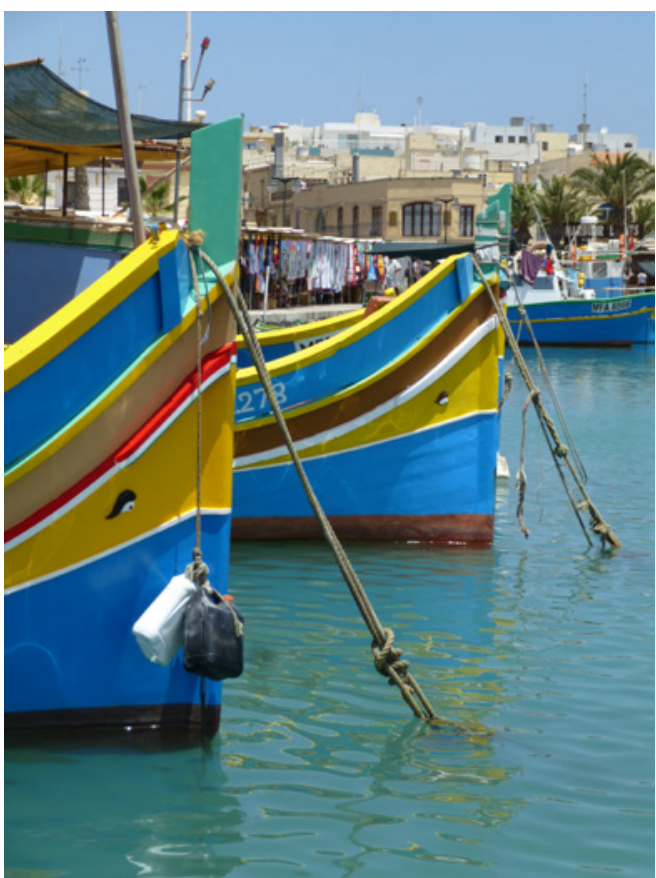

two or three outpatient clinics per week, and weekly interventional and flexible bronchoscopy lists. In addition, the consultants are on call for respiratory medicine for 1 week every month, and the registrar and consultant of that firm take the respiratory consultations during normal working hours, and deal with them accordingly. This is when the firm receives most of its pleural procedure referrals too. Although trainees receive requests for consultations, they are able to discuss patients who have been referred for consultation with their consultant. This system suggests that respiratory medicine can be very busy but it also helps that trainees find a mentor in their consultant and often build a solid working relationship over the course of the year of working together. It also ensures good continuity of care since the firm reviews its inpatients daily and then follows them up at outpatient clinics too. Patients who require regular medical attention become familiar with the firm's doctors.

\section{Out of hours on-calls}

Out of hours on-calls are in general internal medicine, $24 \mathrm{~h}$ long and include carrying the cardiopulmonary resuscitation (CPR) pager. Respiratory cases and noninvasive ventilation patients are admitted to general medicine wards. The on-calls tend to be busy, with reviews of medical admissions and reviews of patients referred from the emergency department of other specialities. Although very tiring, they offer an excellent opportunity to learn and build confidence as a doctor.

\section{Typical day}

A typical day at work starts at about $07: 20 \mathrm{~h}$ by going through the results of the firm's in-patients' tests and identifying any problems that need to be dealt with immediately. Outpatient clinic then starts at $08: 00 \mathrm{~h}$ and usually finishes by $13: 00 \mathrm{~h}$, after which the firm does a ward round of the in-patients. If I happen to be the respiratory medicine on-call doctor that week, then the consultant and myself would receive phone calls throughout the day, and review the referred patients and perform any indicated pleural procedures after the outpatient clinic. On days, I am also on call for general internal medicine and receive calls about general internal medicine problems. These are the days one must rely on having an understanding consultant who is supportive and understands why you may mysteriously disappear from a busy outpatient clinic for an hour or so, only to explain later that you had to rush off to deal with a CPR call.

It is admittedly challenging, especially during the peak winter period when patients are overflowing from the wards. However, it is equally rewarding, and the system of working in teams really helps foster an environment of teamwork and support.

\section{Conflict of interest}

None declared. 